\title{
Detecting High-Refractive-Index Media Using Surface Plasmon Sensor with One-Dimensional Metal Diffraction Grating
}

\author{
Atsushi Motogaito ${ }^{1,3}{ }^{*}$, Shinya Mito1, Hideto Miyake ${ }^{2,3}$, Kazumasa Hiramatsu ${ }^{1,3}$ \\ ${ }^{1}$ Graduate School of Engineering, Mie University, Tsu, Japan \\ ${ }^{2}$ Graduate School of Regional Innovation Studies, Mie University, Tsu, Japan \\ ${ }^{3}$ The Center of Ultimate Technology on nano-Electronics, Mie University, Tsu, Japan \\ Email: "motogaito@elec.mie-u.ac.jp
}

Received 13 June 2016; accepted 15 July 2016; published 18 July 2016

Copyright (C) 2016 by authors and Scientific Research Publishing Inc.

This work is licensed under the Creative Commons Attribution International License (CC BY).

http://creativecommons.org/licenses/by/4.0/

\section{Abstract}

We experimentally detect high-refractive-index media $(n>1.5)$ using a surface plasmon resonance (SPR) sensor with a diffraction grating. While SPR sensors are generally based on the attenuated total reflection method using metal films, here, we focus on a method using a diffraction grating, which can detect relatively higher refractive-index media and is suitable for device miniaturization. In this study, we used the rigorous coupled-wave analysis method to simulate the dependence of the reflectance on an incident angle for media with refractive index values up to 1.700. In the experiment, a medium $(n=1.660-1.700)$ was successfully detected using this grating. Under the conditions of the grating (period: $600 \mathrm{~nm}$, Au thickness: $40 \mathrm{~nm}$ ) using a red laser ( $\lambda: 635$ $\mathrm{nm}$ ), a sharp decline in the reflectance and a rise in the transmittance at certain angles were confirmed, demonstrating the extraordinary transmission enabled by SPR. Because excitation angles changed with changes in the refractive index, we concluded that this method can be applied to sensors that detect high-refractive-index media.

\section{Keywords}

Surface Plasmon Resonance, High-Refractive-Index Media, Diffraction Grating, Extraordinary Transmission

\section{Introduction}

Surface plasmon resonance (SPR) is widely used in various applications such as in the detection of infinitesimally

*Corresponding author.

How to cite this paper: Motogaito, A., Mito, S., Miyake, H. and Hiramatsu, K. (2016) Detecting High-Refractive-Index Media Using Surface Plasmon Sensor with One-Dimensional Metal Diffraction Grating. Optics and Photonics Journal, 6, $164-170$. 
sized materials and high-efficiency photoelectric conversions [1] [2]. Compared to an enzyme-linked immunosorbent assay, it is possible to easily detect a medium from a small amount of samples using sensors. SPR sensors using light were first proposed by Nylander et al. in 1982 [3], and the use of a thin-metal-film-based sensing technique known as the attenuated total reflection method was proposed by Kretchmann [4]. In this method, because resonance results from the use of the total reflection of light, the method is affected by the relation between the refractive index of a target medium and the contact portion of a sensor. For a target medium with a high refractive index ( $n>1.5$ ), total reflection generally becomes impossible owing to a limitation of the incident angle of light; therefore, such a medium cannot be detected. A solution to this problem was demonstrated in a study using a GaP semiconductor, which has a high refractive index of its substrate [5]. In this case, to enable the use of the attenuated total reflection method for the GaP substrate and a thin gold metal film, a prism was used to admit the light into a sensor, and therefore, the detection of a medium with a refractive index of 1.62 was confirmed. However, even for this structure, owing to the high refractive index of the substrate, the detection of a medium with a refractive index greater than 1.62 is impossible because of the limitation of the incident angle. Moreover, for a device involving a prism to admit light into the GaP substrate, the structure of a sensor must be large, hindering device miniaturization.

In this study, we focus on the diffraction grating method using the diffraction grating as the structure of the sensor. In the diffraction grating method, a metal diffraction grating is used instead of a thin metal film and the resonance is caused by the diffraction of light [6]. Because the diffraction grating method does not use total reflection, the limitation of the incident angle is relaxed. An additional advantage of this method is the flexibility of being able to change the detection range and the excitation angle by changing the period and the duty ratio of the grating. Homola et al. investigated the sensitivity of SPR to the grating structure and the amount of processable information, confirming the high information processing capability of diffraction grating structures [7] [8]. In addition, a study of the target localization showed that the differences in the region of the contact between the target medium and the sensor affect the sensitivity [9].

However, previous studies have focused primarily on materials with refractive indices less than 1.5. Many harmful media, such as halogen compounds (e.g., $\mathrm{Br}_{2}: n=1.640$ ) and heavy metal compounds (e.g., $\mathrm{C}_{14} \mathrm{H}_{16} \mathrm{~Pb}: n$ $=1.626$ ), exhibit refractive indices greater than 1.5. The use of surface plasmon sensors for the safe detection of such media is considered advantageous because of their simple operation and ability to detect small amounts of a target. In this study, for the first time, we experimentally demonstrate the detection of a high-refractive-index medium ( $n=1.660-1.700)$ using surface plasmon sensors. To this end, we fabricated a surface plasmon sensor with a metal diffraction grating and evaluated the optical characterization for various materials with refractive indices greater than 1.5 .

\section{Theoretical and Experimental Methods}

\subsection{Numerical Simulations}

We calculated the dependence of the reflectance on the incident angle using the rigorous coupled-wave analysis (RCWA) method for the case of a metal diffraction grating. The simulation model is illustrated in Figure 1, and the simulation conditions were as follows: a metal diffraction grating period of $600 \mathrm{~nm}$, a duty ratio of 0.5 , an Au thickness of $40 \mathrm{~nm}$, a light source wavelength of $635 \mathrm{~nm}$, and polarized light in the TM wave. The refractive index of the glass substrate was $n_{\mathrm{g}}=1.45$ and the dielectric constant of the Au film was $N_{\mathrm{Au}}=0.18-3.51 i$. Four media with refractive indices $n=1.660,1.676,1.686$, and 1.700 were simulated.

\subsection{Experiments}

The metal diffraction grating used in this study was fabricated using electron beam lithography and sputtering on a glass substrate. The resist was applied on a glass substrate and the pattern was formed via irradiation with an electron beam. Then, metal (Au) was sputtered and a one-layer metal diffraction grating was formed via removing the resist with a lift-off process. The metal thickness, period, and duty ratio of the diffraction grating fabricated in this study were $40 \mathrm{~nm}, 600 \mathrm{~nm}$, and 0.5 , respectively. Prior to electron beam lithography, the Au thickness is measured using spectroscopic ellipsometry (UVISEL, HORIBA) and a non-contact film thickness meter (FR-pOrtable, Theta Metrisis). By using various thickness samples, the thickness of Au is determined to be $40 \mathrm{~nm}$. The size of the sample was $1.5 \times 1.5 \mathrm{~mm}^{2}$. The scanning electron microscopy (SEM) image of the surface of the structure in Figure 1 is presented in Figure 2, showing that the sample was fabricated as designed. 


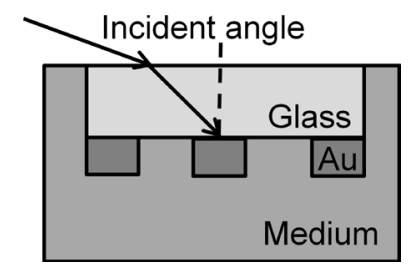

Figure 1. Simulation model of the diffraction grating structure using the RCWA method (period: $600 \mathrm{~nm}$, Au thickness: $40 \mathrm{~nm}$ ).

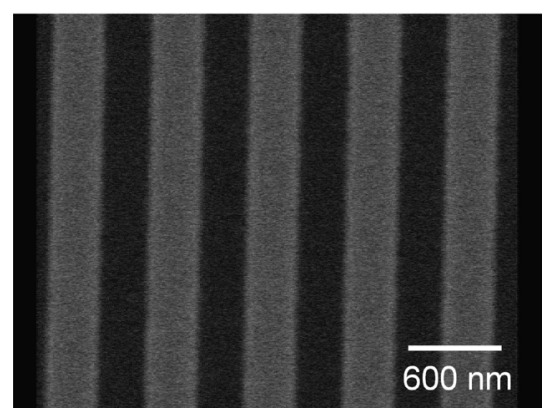

Figure 2. Surface SEM image of the fabricated diffraction grating (period: $600 \mathrm{~nm}$ ).

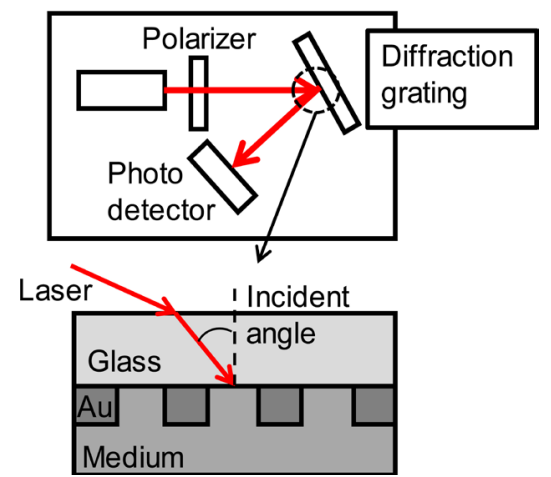

Figure 3. Measurement methods for the dependence of the reflectance on the incident angle (light source: red laser, $\lambda: 635 \mathrm{~nm}$ ).

The method to measure the reflectance is illustrated in Figure 3. A red laser with a wavelength of $635 \mathrm{~nm}$ was used as the light source, and the TM polarized light entered the sample through a polarizer. The liquid medium was placed in a transparent quartz cell, the metal side of the sample was in contact with the liquid, and the light was irradiated from the side of the glass substrate. The reflection intensity was measured for different incident angles of light to investigate the dependence of the reflectance on the incident angle.

\section{Results and Discussion}

\subsection{Theoretical and Experimental Results}

Figure 4 shows a graph of the dependence of the reflectance on the incident angle obtained in our numerical simulations. For all refractive index values, a sharp decline in the reflectance was observed at a certain angle. Based on results from previous studies of surface plasmon sensors, it is thought that this angle is the surface plasmon polariton (SPP) excitation angle [7]. Further inspection of Figure 4 shows that the SPP excitation angles are shifted to higher values with the increasing refractive index of the medium. These results show that the fabricated structure displays the characteristics of an effective sensor because the SPP excitation angles clearly change with minute changes in the refractive index and exhibit a sharp decline in the reflectance.

Figure 5 shows the experimental measurement results of the dependence of the reflectance on the incident 


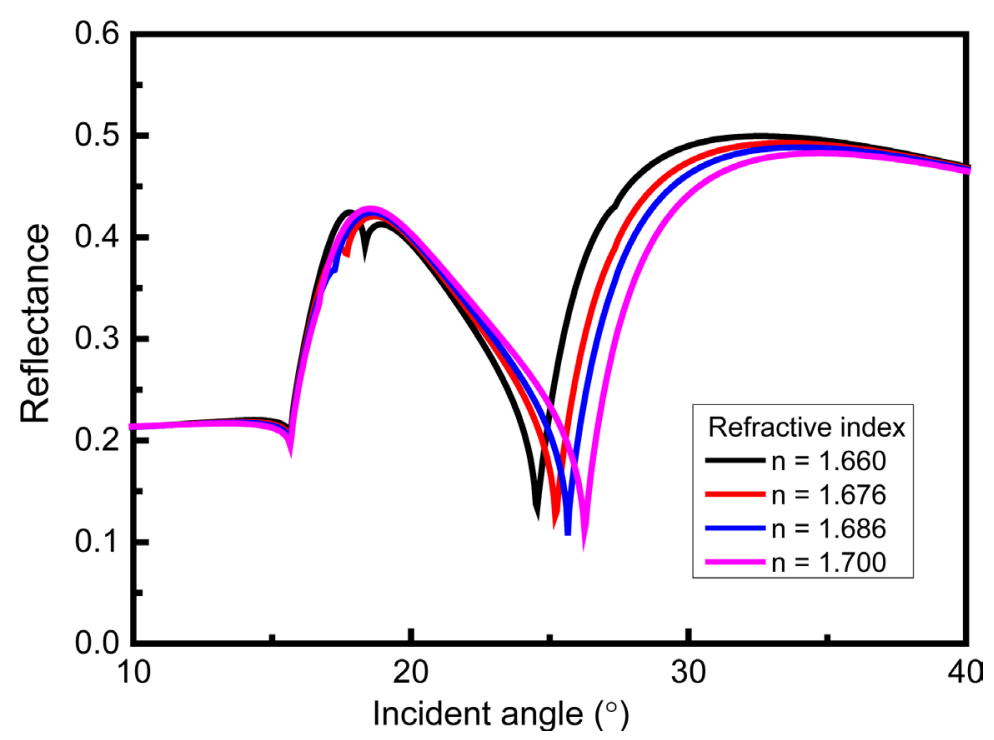

Figure 4. Simulation results for the dependence of the reflectance on the incident angle (media: $\mathrm{n}=1.660,1.676,1.686$, and 1.700).

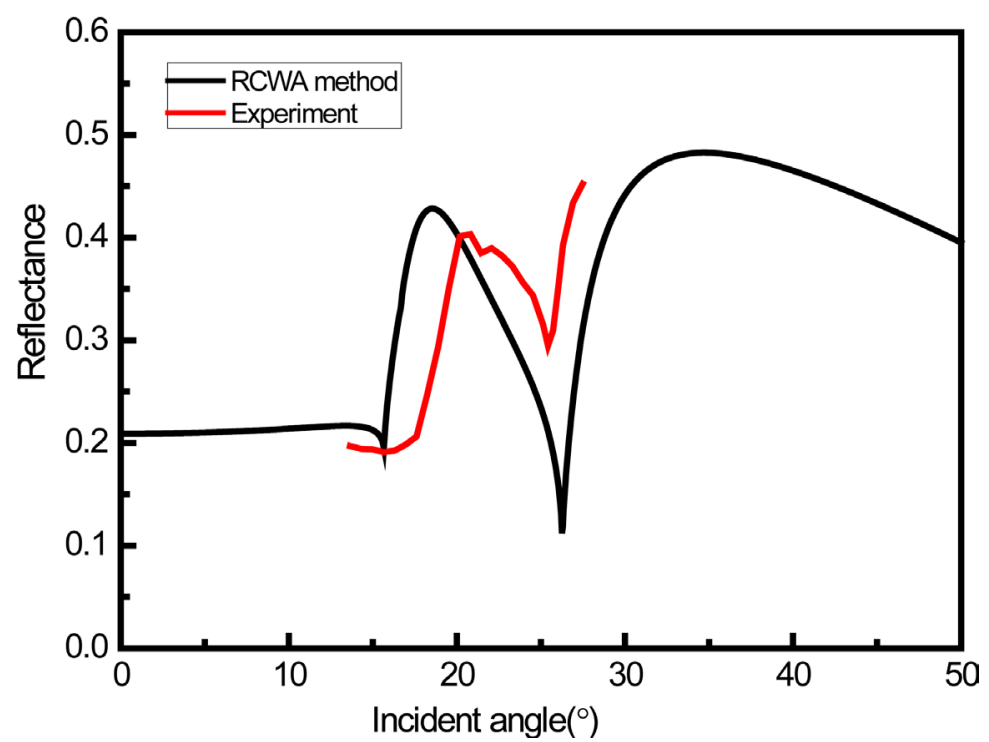

Figure 5. Dependence of the reflectance on the incident angle in the simulation and the experiment (medium: $n=1.700)$.

angle in the case of the refractive index $n=1.700$. Due to the limitation of the measurement system, the experiment is conducted between $15^{\circ}$ and $30^{\circ}$. The simulation results are shown by the black curve and the experimental measurement results are shown by the red curve. Experimentally, a sharp decline in the reflectance was observed at an angle of $25.6^{\circ}$. This value is essentially equal to the excitation angle $\left(26.2^{\circ}\right)$ expected from the simulation. It is speculated that the mismatch between experimental and numerical data is caused by the subtle difference between the simulation model and the actual structure. Moreover, further measurements using three other media showed declines in the reflectance at nearly the same angles as those predicted by the simulations. The four experimentally discovered excitation angles are shown in Figure 6 together with the simulation results. Even though the measured results are shifted slightly to the lower angle side compared to the simulation results, it was confirmed that the excitation angles change with the change in the refractive index. If the simulation model can be close to the actual structure, the simulation results can be improved so as to better match the experimental data. Furthermore, from the results of [5], we can continue to observe this type of match between the experimental data and simulation. 


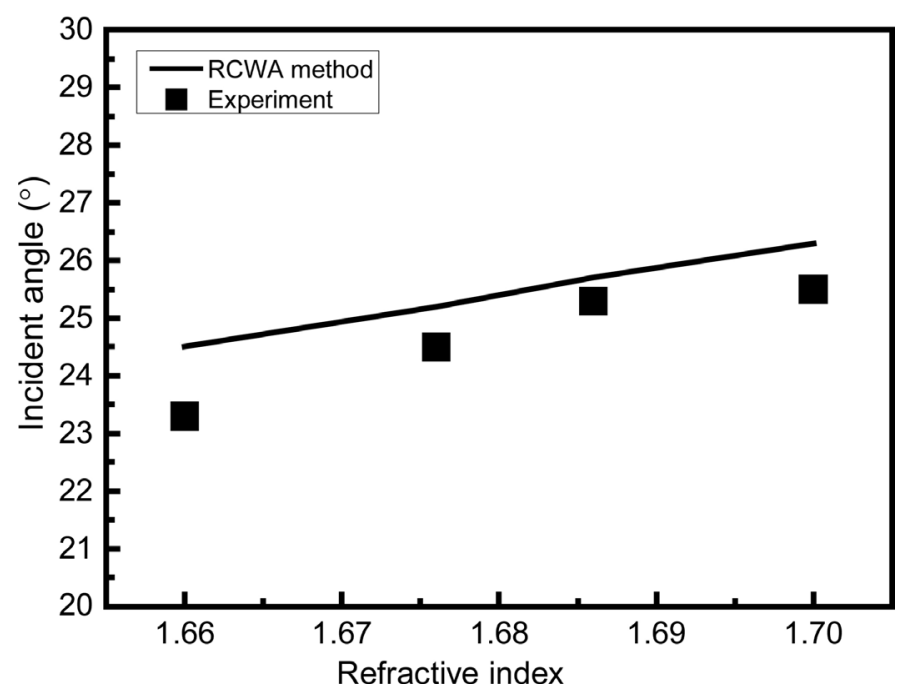

Figure 6. Dependence of the excitation angle on the refractive index in the simulation and in the experiment.

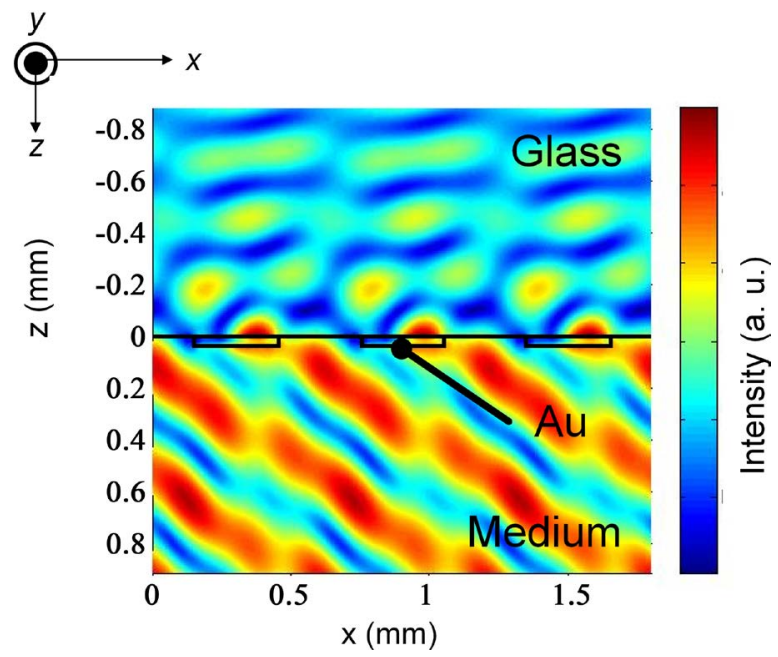

Figure 7. Magnetic field distribution at the excitation angle (medium: $n=1.700$ ).

\subsection{Discussion}

The magnetic field distribution at the SPP excitation angle for a medium with $n=1.700$ is shown in Figure 7 . The incident angle is $25.6^{\circ}$. The magnetic field is concentrated at the interface between the Au and the medium. This is the origin of the surface plasmon polariton. It combines with the transmitted light in the medium, and then, a strong transmitted light can be obtained. An inspection of Figure 7 confirms that the light entering the medium from the glass substrate is transmitted strongly in the medium via the extraordinary transmission by SPR. The dependences of the reflectance and transmittance on the incident angle in the medium with $n=1.700$ are shown in Figure 8, and a sharp decline in the reflection and a sharp increase in the transmittance at the SPP excitation angle are observed. These results suggest that the highly efficient light transmission through the metal diffraction grating is enabled by the coupling of the propagating SPP excited at the interface between the Au and the medium with the light propagating through the medium [10] [11].

\section{Conclusions}

We investigated SPR using a metal diffraction grating as a sensor to detect a high-refractive-index medium using four different materials with refractive indices varying from 1.660 to 1.700 to test our approach. For every medium, it was found that the experimentally obtained excitation angles were very similar to the SPP excitation 


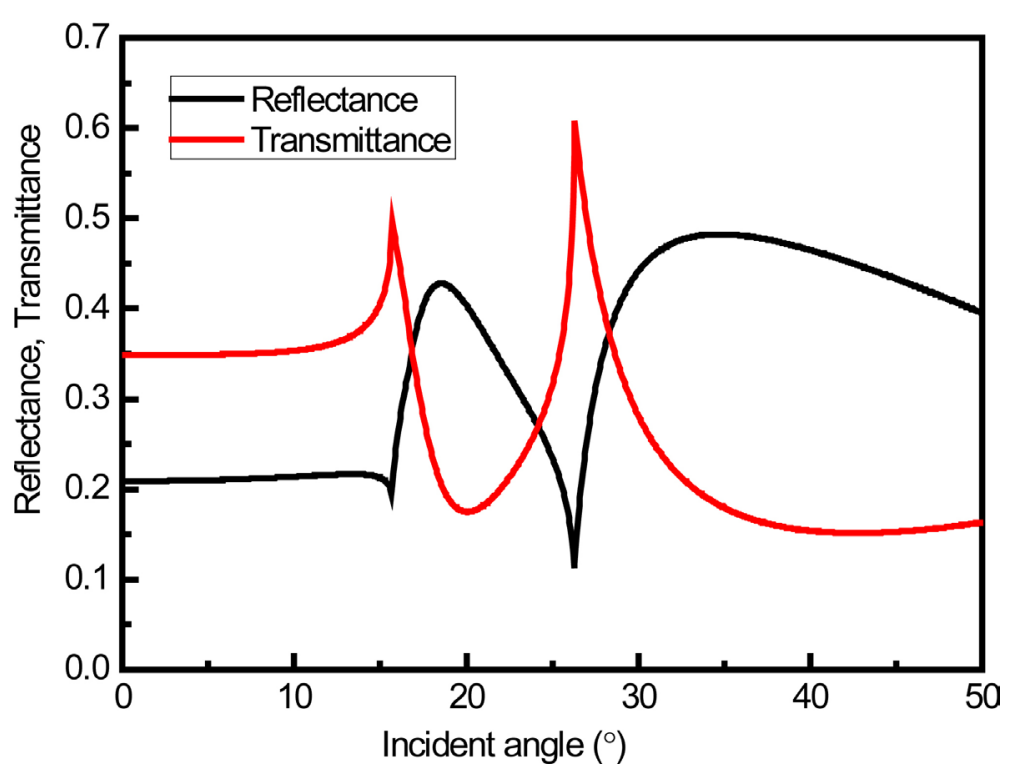

Figure 8. Dependence of the reflectance and transmittance on the incident angle (medium: $\mathrm{n}=1.700$ ).

angles predicted by the numerical simulations. The angular transmittance and magnetic field distribution show that an extraordinary light transmission phenomenon is likely enabled by SPR. Furthermore, it was found that SPP excitation angles shifted to the higher angle side with slight increases in the refractive index, clearly resolving the change in the SPP excitation angle for different materials. Therefore, we conclude that it is possible to detect a medium with a refractive index as high as 1.700 using a SPR sensor with an Au diffraction grating.

\section{Acknowledgements}

This study was supported in part by the Murata Science Foundation and JSPS KAKENHI Grant Numbers 25600090, 26390082, and 15H03556. The authors would like to thank Enago (www.enago.jp) for the English language review.

\section{References}

[1] Hering, K. Cialla, D., Ackermann, K., Dörfer, T., Möller, R., Schneidewind, H., Mattheis, R., Fritzsche, W., Rösch, P. and Popp, J. (2008) SERS: A Versatile Tool in Chemical and Biochemical Diagnostics. Analytical and Bioanalytical Chemistry, 390, 113-124. http://dx.doi.org/10.1007/s00216-007-1667-3

[2] Atwater, H.A. and Polman, A. (2010) Plasmonics for Improved Photovoltaic Devices. Nature Materials, 9, $205-213$. http://dx.doi.org/10.1038/nmat/2629

[3] Nylander, C., Liedberg, B. and Lind, T. (1982/83) Gas Detection by Means of Surface Plasmon Resonance. Sensors and Actuators, 3, 79-88. http://dx.doi.org/10.1016/0250-6874(82)80008-5

[4] Kretschmann, E. (1971) Die Bestimmung optischer Konstanten von Metallen durch Anregung von Oberflächenplasmaschwingungen. Zeitschrift für Physik, 241, 313-324. (In German) http://dx.doi.org/10.1007/BF01395428

[5] Motogaito, A., Nakamura, S., Miyazaki, J., Miyake, H. and Hiramatsu, K. (2015) Using Surface-Plasmon Polariton at the GaP-Au Interface in order to Detect Chemical Species in High-Refractive-Index Media. Optics Communications, 341, 64-68. http://dx.doi.org/10.1016/j.optcom.2014.12.006

[6] Kano, H. and Kawata, S. (1995) Grating-Coupled Surface Plasmon for Measuring the Refractive Index of a Liquid Sample. Japanese Journal of Applied Physics, 34, 331-335. http://dx.doi.org/10.1143/JJAP.34.331

[7] Homola, J., Koudela, I. and Yee, S.S. (1999) Surface Plasmon Resonance Sensors Based on Diffraction Gratings and Prism Couplers: Sensitivity Comparison. Sensors and Actuators B, 54, 16-24. http://dx.doi.org/10.1016/S0925-4005(98)00322-0

[8] Dostálek, J., Homola, J. and Miler, M. (2005) Rich Information Format Surface Plasmon Resonance Biosensor Based on Array of Diffraction Gratings. Sensors and Actuators B, 107, 154-161. http://dx.doi.org/10.1016/j.snb.2004.08.033

[9] Chamtouri, M., Sarker, M., Moreau, J., Besbes, M., Ghalila, H. and Canva, M. (2014) Field Enhancement and Target 
Localization Impact on the Biosensitivity of Nanostructured Plasmonic Sensors. Journal of Optical Society of America B, 31, 1223-1231. http://dx.doi.org/10.1364/JOSAB.31.001223

[10] Ebbesen, T.W., Lezec, H.J., Ghaemi, H.F., Thio, T. and Wolff, P.A. (1998) Extraordinary Optical Transmission through Sub-Wavelength Hole Arrays. Nature, 391, 667-669. http://dx.doi.org/10.1038/35570

[11] Motogaito, A., Morishita, Y., Miyake, H. and Hiramatsu, K. (2015) Extraordinary Optical Transmission Exhibited by Surface Plasmon Polaritons in a Double-Layer Wire Grid Polarizer. Plasmonics, 10, 1657-1662.

http://dx.doi.org./10.1007/s11468-015-9980-8

\section{Submit or recommend next manuscript to SCIRP and we will provide best service for you:}

Accepting pre-submission inquiries through Email, Facebook, LinkedIn, Twitter, etc.

A wide selection of journals (inclusive of 9 subjects, more than 200 journals)

Providing 24-hour high-quality service

User-friendly online submission system

Fair and swift peer-review system

Efficient typesetting and proofreading procedure

Display of the result of downloads and visits, as well as the number of cited articles

Maximum dissemination of your research work

Submit your manuscript at: http://papersubmission.scirp.org/ 